\title{
Highly Sensitive Cardiac Troponin T as a Biomarker of Myocardial Injury in Acyanotic Congenital Heart Disease
}

\author{
Rishika Das ${ }^{1}$, RN Mandal ${ }^{1}$, Anurag Agarwal ${ }^{1 *}$, Sumod Kurian ${ }^{2}$ and Bhawna Mahajan ${ }^{3}$ \\ ${ }^{1}$ Department of Pediatrics, Maulana Azad Medical College, Associated Lok Nayak Hospital, New Delhi, India \\ ${ }^{2}$ Department of Cardiology, GB Pant Institute of Postgraduate Medical Education and Research, New Delhi, India \\ ${ }^{3}$ Department of Biochemistry, GB Pant Institute of Postgraduate Medical Education and Research, New Delhi, India
}

*Corresponding author: Dr. Anurag Agarwal, Department of Pediatrics, Maulana Azad Medical College and Associated Lok Nayak Hospital, New Delhi, India

\begin{abstract}
Background: To evaluate the occurrence of myocardial injury in acyanotic congenital heart disease (ACHD) using highly sensitive cardiac troponin $\mathrm{T}$ (hs-cTnT) as a biomarker.

Methods: An observational cross sectional study was conducted at a tertiary care center over a period of one year (April 2017 to March 2018). Stable children with ACHD aged between 6 months to 5 years were selected as cases and age matched children without ACHD as controls. Echocardiography was done in all the patients with clinical findings of ACHD. A total of 83 children ( 55 cases and 28 controls) were enrolled. The severity of the ACHD was determined by the defect size and the pressure gradient across the defect on echocardiography. Levels of hs-cTnT were measured using electrochemiluminesence assay. These levels were co-related with the defect size and the pressure gradient.
\end{abstract}

Results: The mean levels of hs-cTnT were significantly higher among the cases $(0.044 \mathrm{ng} / \mathrm{ml})$ than the controls $(0.005 \mathrm{ng} / \mathrm{ml}),(p<0.001)$. A positive correlation was noted between the defect size and the levels of hs-cTnT ( $r=$ 0.276 and the $p$ value $=0.042$ ) and an inverse relationship of the levels of hs-cTnT was seen with pressure gradient ( $r$ $=-0.444$ and the $p$ value $=0.001$ ). The development of pulmonary HTN was associated with higher levels of hs-cTnT.

Conclusion: Higher levels of hs-cTnT suggest myocardial injury in children with ACHD and may be useful to stratify children for early surgical intervention before irreversible damage occurs.

\section{Keywords}

Acyanotic congenital heart disease, Myocardial injury, Pulmonary hypertension, Highly sensitive cardiac troponin $\mathrm{T}$

\section{Introduction}

Congenital heart disease (CHD) is defined as a gross structural abnormality of the heart or intrathoracic great vessels that is actually or potentially of functional significance [1]. The burden of CHD in India is enormous because of a very high birth rate. It is estimated that over 180,000 children in India are born with CHD every year [2].

In acyanotic congenital heart disease, anatomical defects are associated with abnormalities in hemodynamic load and neurohormonal activation. This results in myocardial damage which may not be entirely reversible. Biomarkers are important tools which can be used for assessment of the disease and injury to the myocardium [3]. Cardiac troponins are one such biomarker that regulates the interaction between actin and myosin in cardiac myocytes. They are predominantly myofibril bound and only $5-8 \%$ being unbound in the cytosol [4]. They do not occur in extra-cellular space, so their presence in serum is a sensitive marker of cardiac myocytes injury [5]. Therefore, we aimed to evaluate the occurrence of myocardial injury in acyanotic congenital heart disease (ACHD) using highly sensitive cardiac troponin T (hs-cTnT) as a biomarker.

\section{Methods}

This observational cross-sectional study was conducted over a period of one year (April 2017 to March 2018) in the department of Pediatrics, Maulana Azad

Citation: Das R, Mandal RN, Agarwal A, Kurian S, Mahajan B (2020) Highly Sensitive Cardiac Troponin T as a Biomarker of Myocardial Injury in Acyanotic Congenital Heart Disease. Int J Pediatr Res 6:069. doi. org/10.23937/2469-5769/1510069

Accepted: October 12, 2020: Published: October 14, 2020

Copyright: (C) 2020 Das R, et al. This is an open-access article distributed under the terms of the Creative Commons Attribution License, which permits unrestricted use, distribution, and reproduction in any medium, provided the original author and source are credited. 
Medical College and associated Lok Nayak Hospital, New Delhi. The study was conducted in a both inpatient and outpatient setting. After taking ethical clearance from the institute ethics committee and informed consent from the parents/caregivers of the children, a total of 83 children ( 55 cases and 28 age matched healthy controls) between the ages of 6 months to 5 years were enrolled. A study conducted by Hafez, et al. [6] showed the prevalence of myocardial injury in $82.5 \%$ acyanotic congenital heart disease patients. By taking into account $90 \%$ confidence level and $10 \%$ margin of error, the sample size calculated using online sample size calculator [http:// www.raosoft.com/samplesize.html]. Detailed history regarding the age of onset of presentation, duration of symptoms and the presenting complaints was taken and thereafter echocardiography was performed in children with clinical suspicion of ACHD. Children with atrial septal defect (ASD), pulmonary artery hypertension and those taking cardiotoxic drugs were excluded. Sick children and those presenting with congestive cardiac failure were enrolled once they had settled. The presence of a shunt lesion, its direction and size was recorded on echocardiography. Furthermore, the pressure gradient across the defect and the ejection fraction was also determined. Three milliliters of venous blood sample was drawn and collected in a plain vial. The levels of hs-cTnT were measured by electro-chemiluminesence assay using commercially available kits procured from Roche diagnostics India Pvt. Ltd, on Elecsys 2010, cobas e 411 analysers (Manheim, Germany). The measuring range was $0.003 \mathrm{ng} / \mathrm{ml}$ to $10 \mathrm{ng} / \mathrm{ml}$.

\section{Statistical Analysis}

The distribution of the variables was tested with the Shapiro-Wilk test/Kolmogorov Smirnov tests of normality. Group comparisons of the values of skewed data were made with the Mann Whitney test for the two groups. For normally distributed data Student t-test was applied to compare the two groups. Categorical variables were reported as counts and percentages. Group comparisons were made with the Chi-Square test or Fisher's exact test. A $p$ value $<0.05$ was considered significant. Analysis was conducted using IBM SPSS STATISTICS (version 22.0).

\section{Results}

Overall, 83 children between the age of 6 months to 5 years were enrolled amongst which 55 were children with ACHD and 28 were stable controls. The demographic details of the study is provided in Table 1. The median age of presentation of cases was 12 months and 18 months in the controls. The most common presenting symptom among the cases was fast breathing. $80 \%$ of the cases were in CHF at presentation and they were enrolled once they had settled. Most of our cases had severe growth faltering and this could be attributed to inadequate caloric intake as well as increased energy
Table 1: Baseline demographic characteristics.

\begin{tabular}{|l|l|l|}
\hline & Cases $(\mathbf{n}=\mathbf{5 5})$ & Controls $(\mathbf{n}=\mathbf{2 8})$ \\
\hline Median age $(\mathrm{mo})^{*}$ & 12 & 18 \\
\hline Sex $(\mathrm{M}: \mathrm{F})$ & 1.15 & 1.15 \\
\hline WAZ & $-2.99(1.26)$ & $-0.93(1.44)$ \\
\hline HAZ & $-1.46(0.89)$ & $-0.31(0.74)$ \\
\hline $\begin{array}{l}\text { Symptom duration } \\
\text { (days) }\end{array}$ & $2.66(0.86)$ & $7.11(12.20)$ \\
\hline
\end{tabular}

All values except age in mean (SD); WAZ: Weight for age $z$ score; HAZ: height for age $z$ score; ${ }^{*} p=0.338,{ }^{*} p=<0.001$.

Table 2: Comparision of various ACHD.

\begin{tabular}{|l|l|l|}
\hline Mean Parameters & $\begin{array}{l}\text { VSD } \\
\mathbf{N}=\mathbf{4 3}\end{array}$ & $\begin{array}{l}\text { PDA } \\
\mathbf{N}=12\end{array}$ \\
\hline $\begin{array}{l}\text { Hs-cTnT } \\
\text { (ng/ml + mean SD) }\end{array}$ & $0.041(0.09)$ & $0.056(0.13)$ \\
\hline $\begin{array}{l}\text { Defect size } \\
\text { (mm + mean SD) }\end{array}$ & $7.04(2.83)$ & $5.17(1.47)$ \\
\hline $\begin{array}{l}\text { Pressure gradient (mmHg } \\
+ \text { mean SD) }\end{array}$ & $43.86(16.28)$ & $51.16(29.53)$ \\
\hline
\end{tabular}

Values depicted in mean (SD); VSD: Ventricular septal defect; PDA: Patent ductus arteriosus; ${ }^{*} p=0.119$.

requirements caused by increased metabolism.

In our study, $77 \%$ of the cases had VSD and $21 \%$ had PDA. The size across the defect and the pressure gradients were determined on echocardiography (Table 2). Figure 1 shows the mean level of hs-cTnT among the cases was $0.044 \pm 0.101 \mathrm{ng} / \mathrm{ml}$ while among the controls it was $0.005 \pm 0.003 \mathrm{ng} / \mathrm{ml}(p<0.001)$. Overall, hs-cTnT levels were raised in $80.4 \%$ of the cases. Although the levels of hs-cTnT among the various ACHDs were elevated (Table 2), there was no significant statistical difference among them $(p=0.119)$.

These values were co-related with the size of the defect and the pressure gradient in order to assess the severity of the defect. A positive correlation was seen between the defect size and the levels of hs-cTnT ( $r=$ 0.276 and $p=0.042$ ) (Figure 2) whereas an inverse relationship was obtained with pressure gradient and defect size $(r=-0.444$ and $p=0.001)$ (Figure 3$)$.

\section{Discussion}

In our study myocardial injury was evidenced by higher levels of hs-cTnT in $80.4 \%$ of the cases. A study by Hafez, et al. [6] in Egypt elicited the evidence of myocardial injury in unoperated congenital heart disease. Increased Cardiac troponin-I was found in 64 out of 80 (80\%) children with CHD. In a similar study by Sugimoto, et al. [7] in Japan, hs-cTnl in the VSD group was significantly higher than those in the ASD group as well as the healthy group. They speculated that the ventricular pressure overload and volume overload in children with ACHD synergistically induces myocardial injury. 


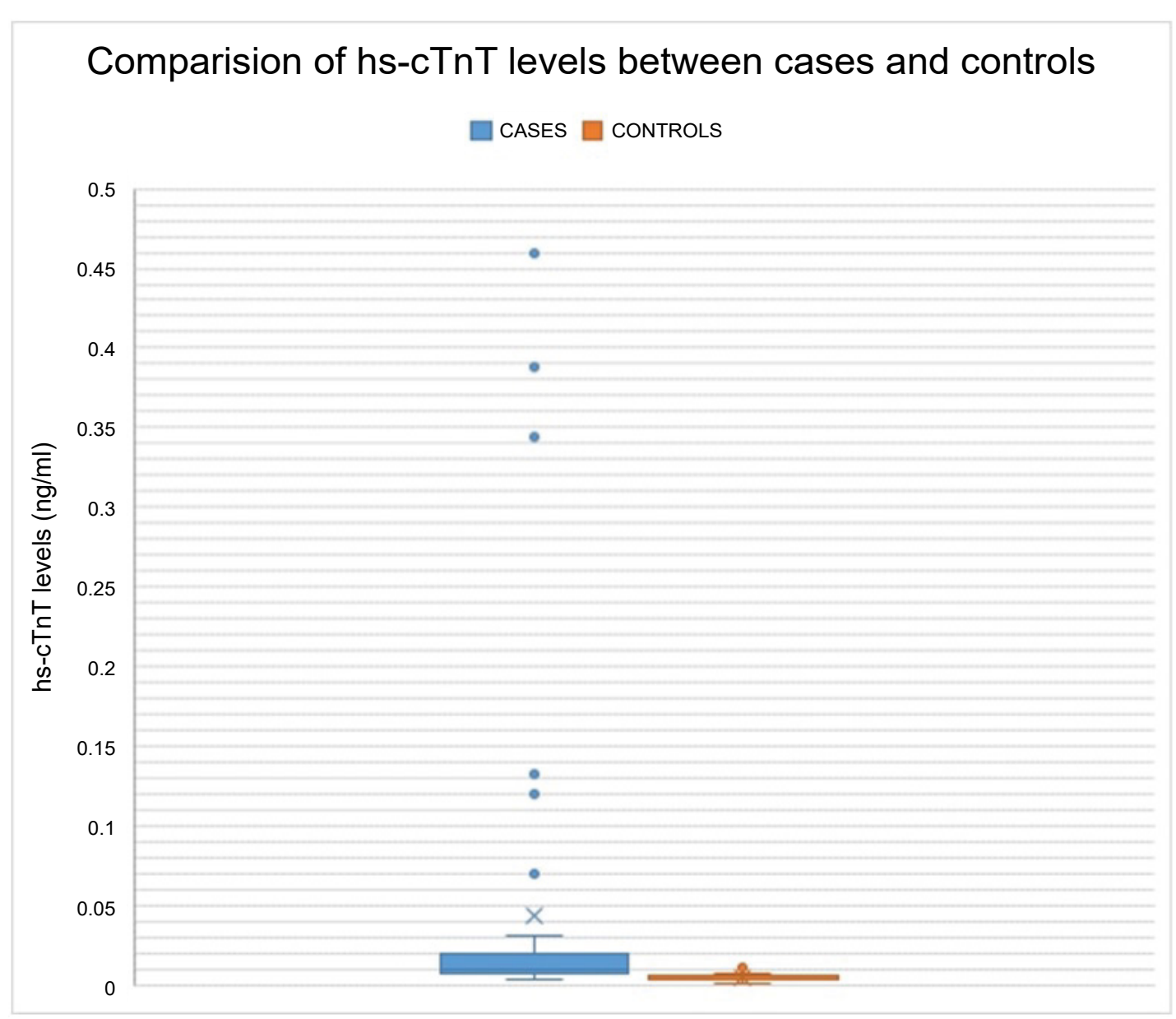

Figure 1: Mean hs-cTnT levels between cases and controls.

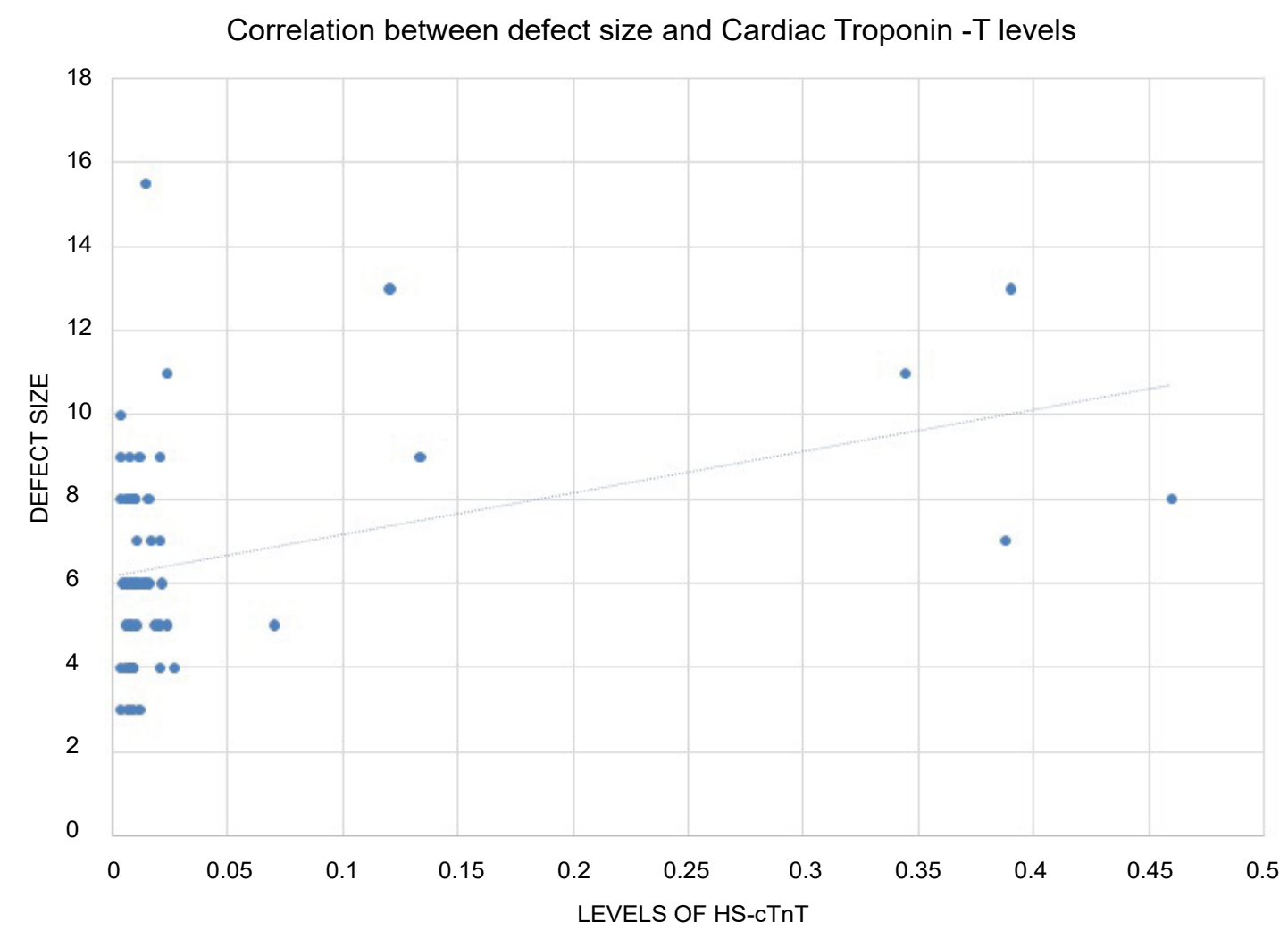

Figure 2: Relationship between Defect Size and Hs-cTnT. It shows positive correlation between the defect size and the levels of hs-cTnT. With increase in size of the defect of ACHD the levels of Cardiac troponin-T show an increasing trend. 


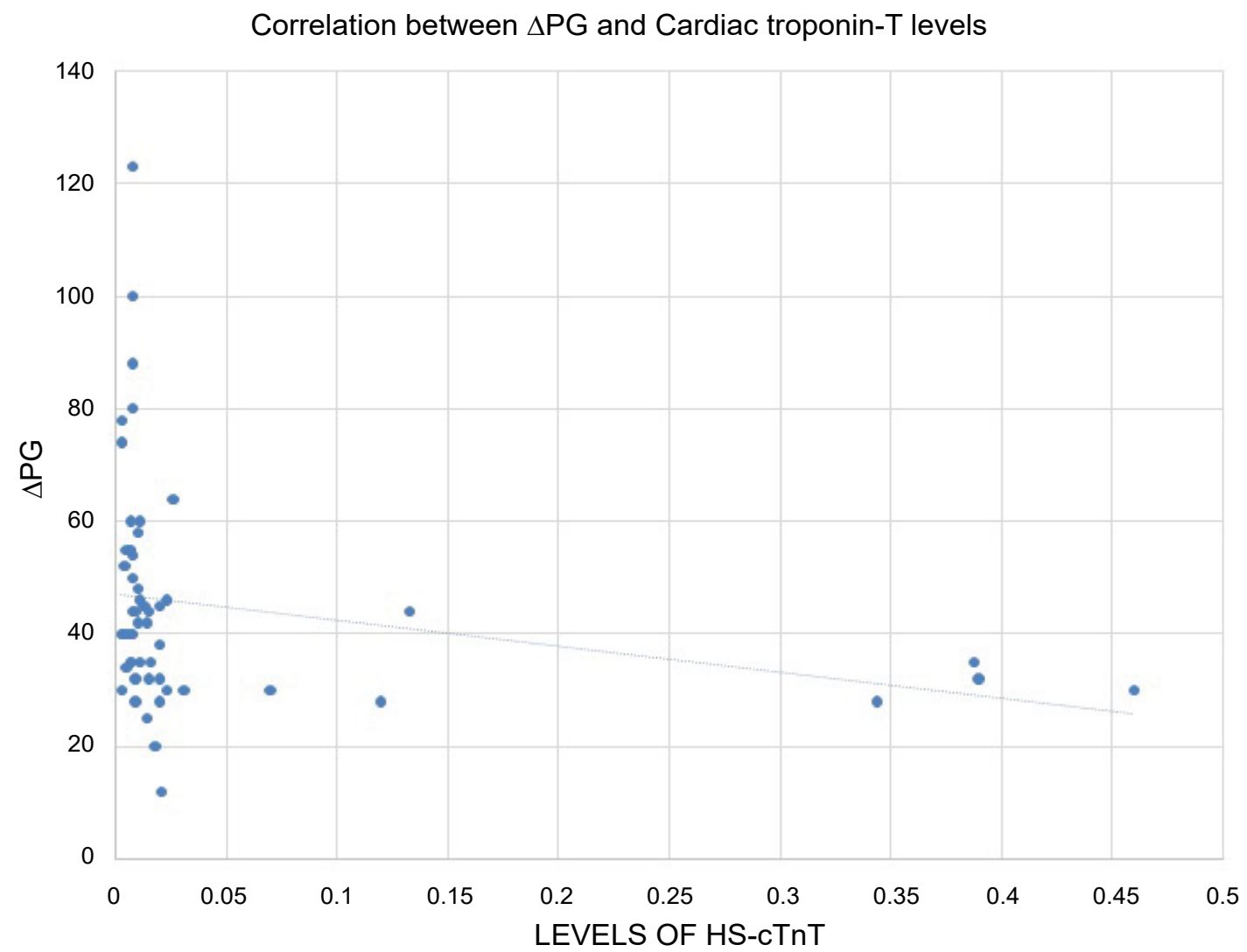

Figure 3: Relationship between Pressure Gradient and Hs-CTnT. It depicts the relationship of pressure gradient with the levels of hs-cTnT. There is an inverse relationship of the level of hs-cTnT with pressure gradient. Hs-cTnT levels show a declining trend at higher pressure gradients. The relation between them is statistically significant.

Tsutamoto, et al. [8] regarded cardiac troponins as the gold standard markers for myocardial injury. Elevation of BNP levels in congenital heart disease is well known. Abiko, et al. [9] showed that the serum concentration of hs-cTnT had significantly correlated with BNP levels. These levels can however be elevated in many non-coronary artery-related conditions like sepsis, septic shock, hypovolemia, heart failure and atrial fibrillation or other tachyarrhythmias. Although these studies have used hs-cTnl, Trevisanuto [10] explained that the sensitivity of both troponins in the identification of myocardial damage is clinically almost equal. Unlike CTnT assay which has only one manufacturer, there are a number of cTnl assays available in the market but they lack standardization.

Eindhoven, et al. [11] claimed that hs-cTnT was modestly elevated in almost $10 \%$ of stable ACHD patients in an outpatient-clinic setting during routine check-up. Therefore, he stated that all ACHD patients have some degree of heart failure and these levels may be useful to monitor ventricular function and further deterioration.

The positive correlation of hs-cTnT levels with the defect size reflect the burden of injury over the myocardium. On the other hand, an inverse relationship with pressure gradient can be explained by the fact that severe ACHD would lead to pulmonary vascular changes and eventually development of PAH. This corroborated with a study conducted by Garg, et al. [12].
The major limitation of the study was that the sample size was relatively small in comparison to the general population of ACHD. As it was a cross-sectional study, these children were not followed up and hence the levels of hs-cTnT in case they underwent surgery are not known.

\section{Conclusion}

We conclude that significant myocardial injury occurs in children with ACHD. Surgical correction of CHD is delayed due to poor nutritional status as well as due to limited resources. This leads to prolonged exposure of cardiac muscle to pressure and volume overloads. Biomarkers like hs-cTnT can predict the progression of the pathological condition. In a country like India where resources are limited, it is important to stratify children with higher levels of hs-cTnT, so that they can be prioritized and taken for surgical intervention early.

\section{Conflict of Interest}

There are no conflicts of interests in this study.

\section{Key Message}

Considerable amount of volume and pressure overload occurs in ACHD due to left to right shunts. These shunts induce significant myocardial injury which can be detected by elevated levels of hs-cTnT.

\section{References}

1. Mitchell SC, Korones SB, Berendes HW (1971) Congenital 
heart disease in 56,109 births. Incidence and natural history. Circulation 43: 323-332.

2. Saxena A (2005) Congenital heart disease in India: A status report. Indian J Pediatr 72: 595-598.

3. Miyamoto K, Takeuchi D, Inai K, Shinohara T, Nakanishi $\mathrm{T}$ (2016) Prognostic value of multiple biomarkers for cardiovascular mortality in adult congenital heart disease: Comparisons of single/two-ventricle physiology, and systemic morphologically right/left ventricles. Heart Vessels 31: 1834-1847.

4. Bleier J, Vorderwinkler KP, Falkensammer J, Peter M, Dapunt O, et al. (1998) Different intracellular compartmentations of cardiac troponins and myosin heavy chains: A causal connection to their different early release after myocardial damage. Clin Chem 44: 1912-1918.

5. B Solnica (2004) Cardiac troponins. Medycyna Praktyczna 10: $133-136$.

6. Hafez MO, Morsy SM, Mahfoz RA, Ali AR (2015) Myocardial Injury in Children with Unoperated Congenital Heart Diseases. Cardiol Res Pract 2015: 104818.

7. Sugimoto M, Ota K, Kajihama A, Nakau K, Manabe H, et al. (2011) Volume overload and pressure overload due to left- to-right shunt-induced myocardial injury- Evaluation using a highly sensitive cardiac Troponin-I assay in children with congenital heart disease. Circ J 75: 2213-2219.

8. T Tsutamoto, C Kawahara, K Nishiyama, Yamaji M, Fujii $M$, et al. (2010) Prognostic role of highly sensitive cardiac troponin I in patients with systolic heart failure. American Heart Journal 159: 63-67.

9. Abiko M, Inai K, Shimada E, Seiji Asagai, Toshio Nakanishi (2018) The prognostic value of high sensitivity cardiac troponin $\mathrm{T}$ in patients with congenital heart disease. J Cardiol 71: 389-393.

10. Trevisanuto D, Pitton M, Altinier S, Zaniotto M, M Plebani, et al. (2003) Cardiac troponin I, cardiac troponis T and creatine kinase MB concentrations in umbilical cord blood of healthy term neonates. Acta Paediatr 92: 1463-1467.

11. Eindhoven JA, Roos-Hesselink JW, van den Bosch AE, Kardys I, Cheng JM, et al. (2015) High-sensitive troponin-T in adult congenital heart disease. Int $\mathrm{J}$ Cardiol 184: 405411.

12. Garg A. Shrivastava S, Radhakrishnan S, V Dev, A Saxena (1990) Doppler assessment of interventricular pressure gradientacross isolated ventricular septal defect. Clin Cardiol 13: 717-721. 